'Clínica IRAM. Santiago, Chile. 2Programa Radioterapia Oncológica Universidad Diego Portales. Santiago, Chile.

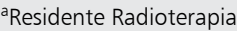
Oncológica Universidad Diego Portales - Clínica IRAM.

Trabajo no recibió financiamiento. Los autores declaran no tener conflictos de interes.

Recibido el 10 de abril de 2019, aceptado el 16 de octubre de 2019

Correspondencia:

Dra. Natalia Jara O. Américo Vespucio Norte 1314, Vitacura. Santiago, Chile. natalia.jara@iram.cl

\section{Fibroxantoma atípico. Caso clínico}

\author{
NATALIA JARA ${ }^{a}$, SEBASTIÁN SOLÉ Z.,2
}

\section{Scalp atypical fibroxanthoma. Report of one case}

Atypical Fibroxanthoma is an unusual dermal mesenchymal tumor. It especially affects older adults and occurs in areas of sun exposure. We report a 75 years old male with a history of sun exposure without using a hat presenting with a scalp nodule. An incisional biopsy showed an atypical fibroxantoma. In a new surgical procedure, the tumor was completely excised. The tumor relapsed in two occasions after subsequent excisions and the patient was treated with adjuvant radiotherapy avoiding new relapses.

(Rev Med Chile 2019; 147: 1346-1349)

Key words: Poroma; Radiotherapy; Scalp.
$\mathrm{E}$ 1 Fibroxantoma Atípico (FXA) es un tumor del mesénquima dérmico poco conocido, descrito en 1961 por Helwing ${ }^{1}$. Afecta a adultos mayores y se presenta en zonas de exposición solar. Actualmente, se sabe que tiene un grado de malignidad intermedio por su capacidad de recidivar y producir metástasis ${ }^{2}$. Ya que es una patología poco conocida, habitualmente no se tiene presente en la practica clínica, lo que afecta el resultado oncológico de estos pacientes. Si consideramos el envejecimiento de la población y que el cáncer de piel no melanoma es la patología oncológica más frecuente, resulta relevante tener presente esta patología dentro de los diagnósticos diferenciales al momento de enfrentar una lesión en piel.

\section{Caso clínico}

Paciente de 75 años sin antecedentes relevantes. Refiere exposición solar sobre el cuero cabelludo durante 30 años diariamente (alopecia), sin uso de sombrero ni bloqueador solar. Consulta por aumento de volumen nodular en región de cuero cabelludo parietal izquierdo progresivo.

Se realizó biopsia incisional el 15.7.14 de nódulo cutáneo fijo no sensible. El informe de anatomía patológica indica tumor maligno fusocelular con compromiso del borde lateral y profundo. Estudio inmunohistoquímico muestra ser negativo para citoqueratina, negativo para $\mathrm{CD} 34$, negativo para actina, negativo para $S 100$, positivo para KP1-PGM1. Los hallazgos de microscopía en asociación a los de inmunoperoxidasa corresponden a un Fibroxantoma Atípico. Se revisa anatomía patológica en otro laboratorio estando de acuerdo con el diagnóstico. El 31.7.14 se hace ampliación quirúrgica (biopsia escicional) que demuestra tumor residual compatible con fibroxantoma atípico resecado con bordes negativos. Figura 1 imágenes de la biopsia del paciente.

En control de seguimiento a los 2 meses de la cirugía presenta recidiva local. Se hace nueva biopsia escicional el 28.10.14. La anatomía patológica muestra una recidiva del fibroxantoma atípico. Tiene una primera consulta en radioterapia el 19.11.14 donde se desestima la necesidad de tratamiento adyuvante.

En control de seguimiento a los 2 meses de la cirugía vuelve a presentar recidiva, en este caso multifocal con 4 lesiones nodulares no sensibles en cuero cabelludo bilateral. Nueva biopsia escicional el 5.2.15 de lesión mayor vuelve a demostrar recidiva local por fibroxantoma atípico.

Se decide biopsia excisional de todas las lesiones apareciendo 2 nuevas antes de la cirugía que se efectuó el 6.3.15. La anatomía patológica confirma 


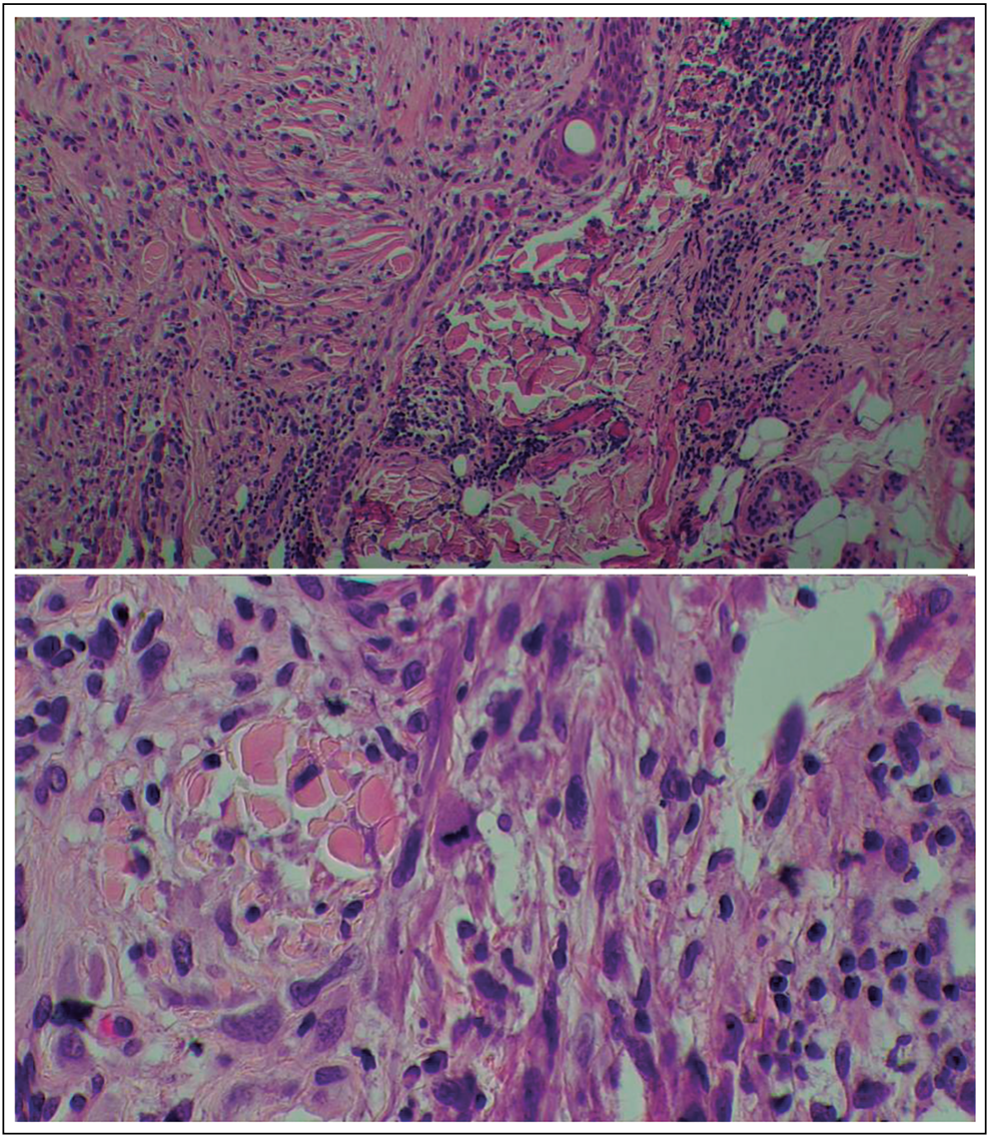

Figura 1. Histología de Fibroxantoma Atípico con tinción de hematoxilina eosina. A: Imagen de 100x de aumento. Se muestra una lesión en dermis profunda de células fusadas y fibrohistiocíticas pleomórficas. B: Imagen de 400x de aumento. Se muestran atipias nucleares y mitosis en el centro de la imagen. Características de malignidad.
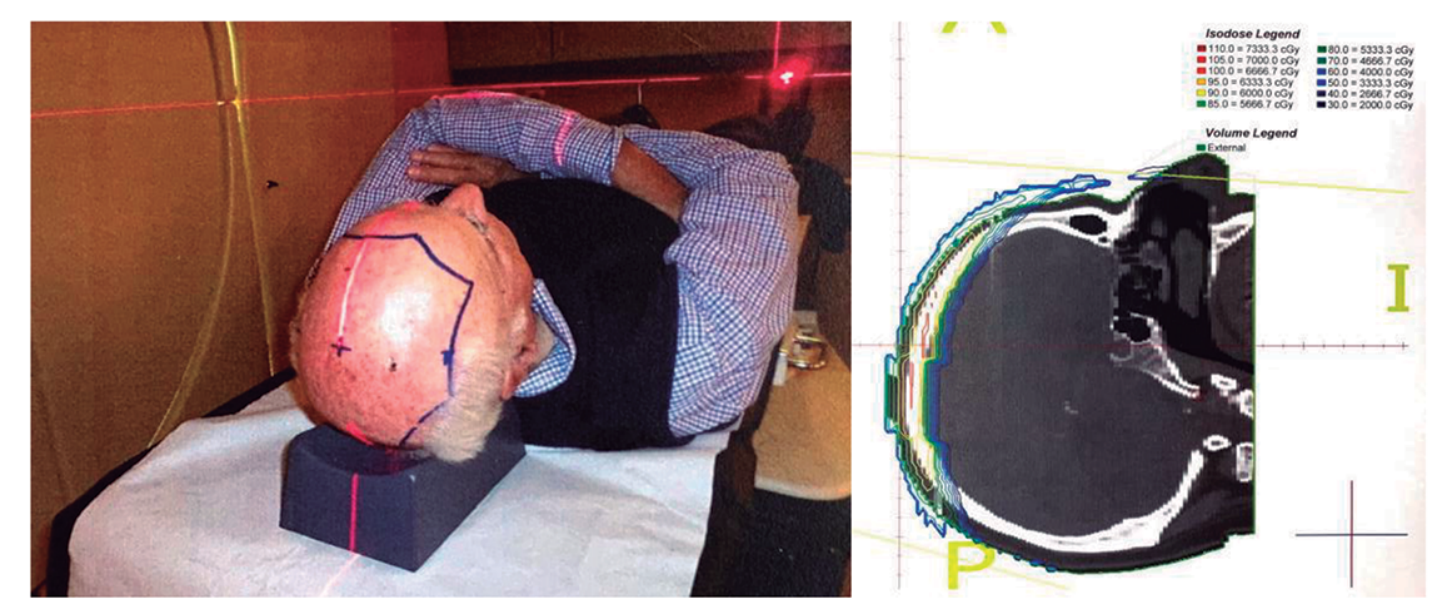

Figura 2. Posicionamiento y dosimetría del tratamiento de radioterapia con electrones. A: Se muestra la zona de tratamiento y posición del paciente en el equipo. B: Se indica la distribución de la dosis, destaca que la dosis de radiación solo es entregada superficialmente, sin afectar estructuras profundas. 


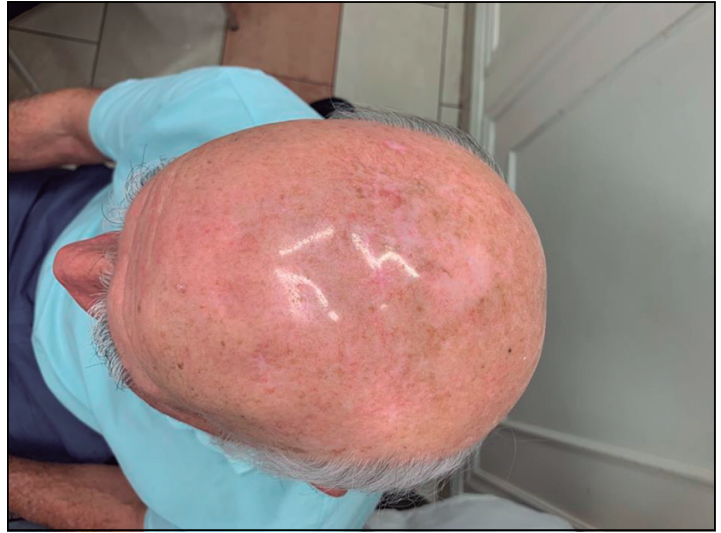

Figura 3. Zona de tratamiento en el control de seguimiento a los 47 meses.

5 lesiones compatibles con fibroxantoma atípico en piel con foto daño grave.

Por las 2 recidivas precoces siendo la última multifocal se decide efectuar tratamiento con radioterapia adyuvante sobre cuero cabelludo 60 Gy en 30 fracciones con electrones de $6 \mathrm{MeV}$. En la Figura 2 posicionamiento y dosimetría de la radioterapia. El tratamiento fue realizado entre el 26.3.15 y el 12.5.15 con buena tolerancia, solo presentando eritema moderado en cuero cabelludo, sin toxicidad severa.

El 19.3.19 acude a control con un seguimiento de 47 meses desde la última sesión de radioterapia. En buenas condiciones, haciendo vida normal. Sin toxicidad crónica. Quedando al examen físico solo las cicatrices planas de sus resecciones, sin nuevas lesiones sospechosas de malignidad. En la Figura 3 muestra la zona de tratamiento en el último control.

\section{Discusión}

El FXA se manifiesta como un tumor solitario exofítico con una tasa de crecimiento rápido. $\mathrm{Su}$ diámetro no excede los $2 \mathrm{~cm}$ en la mayoría de los casos. Es de consistencia firme, no doloroso, de coloración marrón a rojizo, frecuentemente ulcerado. Se localiza en zonas de elastosis solar como cabeza y cuello en $80 \%$ de los casos y ocasionalmente se ha localizado en extremidades inferiores o tronco. Se estima una prevalencia de
0,24\% según un estudio que solo evalúa tumores raros de una cohorte de 42.279 pacientes tratados con cirugía de Mohs ${ }^{3}$.

Se presenta entre los 70 y 80 años afectando a hombres con mayor frecuencia ${ }^{4}$ La patogenia del FXA presenta mutación de P53 inducida por radiación UV, por lo que la exposición solar tiene un rol preponderante ${ }^{5}$.

Histopatológicamente el FXA está compuesto por una mezcla de células fusadas y células fibrohistiocíticas pleomórficas, la imunohistoquímica es positiva para vimentina, CD10, P53 y negativa para citoqueratina, S-100, CD34, ERG, desmina, actina y $\mathrm{H}$-caldesmina ${ }^{6}$.

En una revisión australiana del manejo del $\mathrm{FXA}^{7}$ el tratamiento más utilizado es la resección local, la radioterapia es el principal tratamiento adyuvante para este tumor. La tasa de recurrencia es $4,6 \%$ y el riesgo de metástasis es $0,95 \%$, siendo los sitios más frecuentes los ganglios linfáticos regionales, parótida y pulmón. La mortalidad específica por enfermedad es muy poco frecuente. En pacientes inmunosuprimidos las tasas de recurrencias y metástasis alcanzan $16 \%$ y $6,4 \%$, respectivamente.

Los márgenes quirúrgicos comprometidos se asocian a mayor riesgo de recidiva y metástasis. Se debe considerar realizar linfonodo centinela en casos de tumores con extensión a planos profundos por el riesgo de metástasis ${ }^{8}$. La radioterapia está reservada para lesiones que no pueden ser resecadas o con resección insuficiente. Habitualmente se utilizan dosis de $60-65 \mathrm{~Gy}^{4}$.

Los controles de seguimiento deben realizarse cada 3 a 6 meses en los 3 primeros años, en especial en los pacientes de mayor riesgo. La inspección, palpación de piel y ganglios linfáticos, junto con ecografía ganglionar están recomendadas ${ }^{4}$.

Agradecimientos: Queremos agradecer al Dr. Arturo Espinoza, anatomopatólogo, director medico de CITOLAB que gentilmente nos facilitó las imágenes del estudio histológico del paciente.

\section{Referencias}

1. Helwig EB. Tumor seminar proceedings of the 18th annual tumor seminar of San Antonio Society of Pathologists, 1961: Atypical Fibroxanthoma. Tex J Med 1963; 59: 664-7. 
2. Iorizzo Iii LJ, Brown MD. Atypical Fibroxanthoma: a review of the literature. Dermatol Surg 2011; 37: 146-57.

3. Anderson HL, Joseph AK. A pilot feasibility study of a rare skin tumor database. Dermatol Surg 2007; 33: 6936.

4. Ziemer M. Atypical Fibroxanthoma. J Dtsch Dermatol Ges 2012; 10 (8): 537-50.

5. DeiTos AP, Maestro R, Doglioni C, Gasparotto D, Boiocchi M, Laurino L, Fletcher CD. Ultraviolet-induced p53 mutations in atypical fibroxanthoma. Am J Pathol 1994; 145 (1): 11-7.
6. Mentzel T, Requena L, Brenn T. Atypical Fibrixanthoma Revisited. Surgical Pathology 2017; 10 (2): 319-35.

7. Polez M, Sebaratnam DF, Fernández-Peñas P. Atypical fibroxanthoma management: Recurrence, metástasis and disease-specific death. Australas J Dermatol 2018; 59 (1): 10-25.

8. Love WE, Schmitt AR, Bordeaux JS. Management of unusual cutaneous malignancies: Atypical fibroxanthoma, malignant fibrous histiocytoma, sebaceous carcinoma, extramammary Paget disease. Dermatol Clin 2011; 29: 201-16. 